\title{
Enhancing the Energy Resolution of a Singles Photon STJ Spectrometer Using Diffusion Engineering
}

\author{
Veronica Savu, Luigi Frunzio, and Daniel E. Prober, Member, IEEE
}

\begin{abstract}
Diffusion engineering has been proposed as an approach to increase the collected charge and energy resolution of a single-photon superconducting tunnel junction (STJ) spectrometer. We present new experimental results confirming this approach. When a photon of energy $E$ is absorbed in a superconductor with energy gap $\Delta$, it creates $N$ initial quasiparticles, with $N \approx 0.6(E / \Delta)$. Their charge, upon tunneling, is equal to $\mathrm{Q}=p e N$, with $p=1$ for single tunneling across the voltage biased STJ. The output charge can be amplified by backtunneling, with $p>1$, if the quasiparticles are confined around the junction. This charge multiplication is proportional to the confinement time. Previous work used higher gap superconductors for confinement. In this work, the counterelectrode is terminated by a long, narrow wire made of the same material. We find $p>1$ due to the slow out-diffusion of the quasiparticles down the wire. The wire dimensions and diffusion constant were chosen to engineer the backtunneling multiplication. For large backtunneling, the signal-to-noise of our spectrometer is increased.
\end{abstract}

Index Terms-Backtunneling, optical spectrometer, single photon detector, superconducting tunnel junctions.

\section{INTRODUCTION}

$\mathbf{T}$ HE ADVANTAGE of superconductors over semiconductors in low-energy, single-photon detection is the three-orders-of-magnitude smaller energy gap, on the order of $\mathrm{meV}$. The collected charge created by a visible photon is thus a thousand times larger in a superconductor device. Yet, with available amplifiers, it is difficult to read out even a charge of $10^{3}$ electrons in a short pulse from a cold detector. The backtunneling method can significantly increase this charge.

A superconducting tunnel junction (STJ) consists of two electrodes separated by a thin insulating layer. A photon absorbed in the superconductor breaks Cooper pairs and creates excess quasiparticles, which tunnel across the voltage-biased junction. The charge obtained from integrating the current pulse is a measure of the energy deposited in the superconductor by the photon. The energy resolution of an STJ is limited by the superconducting energy gap $\Delta$ of the material, processes related to the device geometry, and the background and electronic noise. By confining the quasiparticles around the junction area, they tunnel and back-tunnel, effectively multiplying the initially created charge. The advantage of using backtunneling devices is the increase of the signal-to-noise ratio.

Manuscript received August 28, 2006. This work was supported in part by NASA, NASA-GSFC, and NSF-AST.

V. Savu, L. Frunzio, and D. E. Prober are with the Departments of Physics and Applied Physics, Yale University, New Haven, CT 06520 USA (e-mail: veronica.savu@yale.edu; luigi.frunzio@yale.edu; daniel.prober@yale.edu).

Color versions of one or more of the figures in this paper are available online at http://ieeexplore.iee.org.

Digital Object Identifier 10.1109/TASC.2007.898612

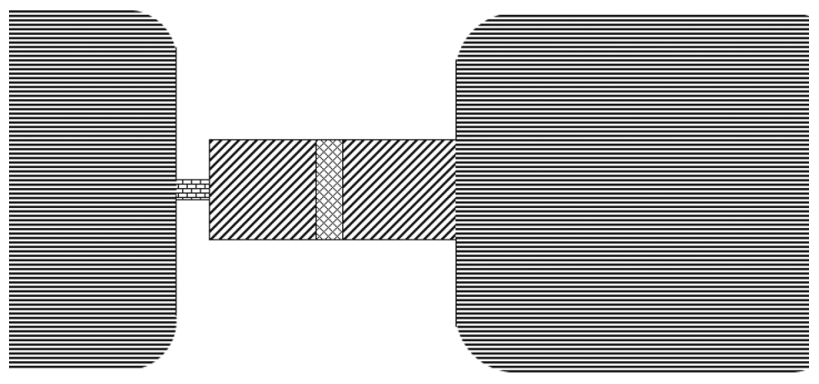

(a)

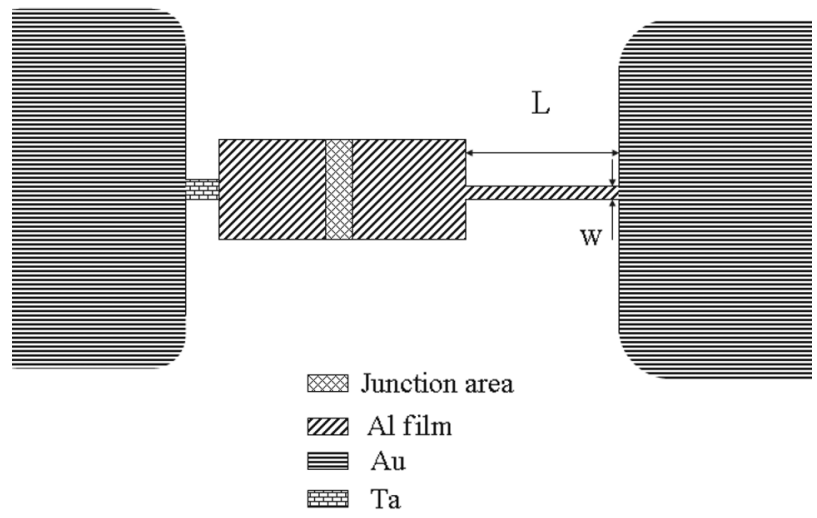

(b)

Fig. 1. Top view of single-tunnelling and diffusion-engineered devices design. a) Single-tunnel device; b) diffusion-engineered device (not to scale).

Astronomy has already benefited from the use of STJs used as single photon spectrometers. The photon's energy (color) as well as its arrival time can be recorded, at a relatively high counting rate. Thus, transient weak signals from distant galaxies can be explored, such as visible light from pulsars and variable stars. The change in brightness of several spectral channels can be recorded in parallel, on millisecond to microsecond timescales [1].

In biology, measurement of fluorescent spectra at the singlephoton level is a challenging issue. For imaging low intensity fluorescent specimens, avalanche photodiodes (APDs) are usually used. For obtaining spectral information, an APD has to be used with a set of narrow band filters. Every time the band filter is changed one can record data at a specific wavelength. For studying multiple chromophores, the sample has to be scanned multiple times, increasing its probability to photobleach. Dispersive gratings on the other hand have as many as 32 energy channels [2], but they are read sequentially with only one PMT. Simultaneous read-out of all the channels would require a high level of experimental complexity.

Using single-tunneling devices with lateral geometries (Fig. 1), energy resolutions of $2.15 \mathrm{eV}$ have been achieved at 

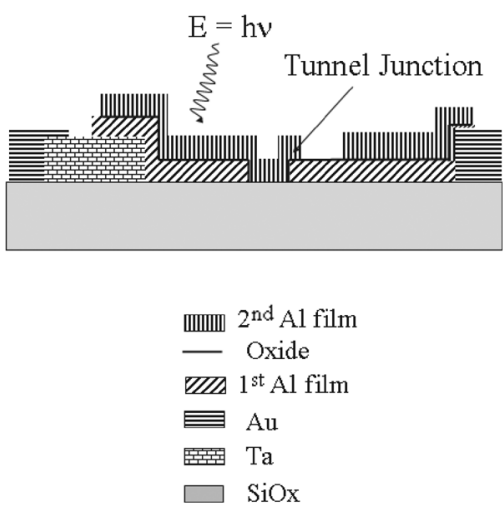

Fig. 2. Side view of a single-tunnel device.

$3.68 \mathrm{eV}$ [3]. Using vertical geometries and strong backtunneling enabled energy resolving powers of up to 25 for $2.5 \mathrm{eV}$ photons [4] $(E / \Delta E$, with $E$ the photon energy and $\Delta E$ the energy full width half maximum). In this paper we present measurements of optimized STJs for optical photons. We obtain an energy resolution of $1.5 \mathrm{eV}$ at $3.68 \mathrm{eV}$ using a diffusion-engineered device. We discuss our results and propose solutions for further improving our device resolution. While our energy resolution is only moderate, this work demonstrates the diffusion-engineered concept.

\section{EXPERIMENTAL RESULTS}

We start the fabrication of our devices by dc-sputtering $70 \mathrm{~nm}$ of Ta on a hot wafer. We pattern it using ion-miling so that for each future device we have a $5 \times 5 \mu \mathrm{m}^{2}$ square continued with a $3 \times 1 \mu \mathrm{m}^{2}$ rectangle. The $100 \mathrm{~nm}$ thick Au contact pads are deposited next, overlapping with the Ta square for good metallic contact. We deposit $\mathrm{Au}$ around the devices to enhance photon reflectivity away from the substrate. The $1 \times 5 \mu \mathrm{m}^{2} \mathrm{Al} / \mathrm{Al}$-oxide/Al tunnel junction is fabricated using the Dolan-bridge deposition technique [5] using a suspended resist bridge. The $50 \mathrm{~nm}$ first $\mathrm{Al}$ layer is deposited at a $40^{\circ}$ angle from the normal to the substrate and the second $70 \mathrm{~nm}$ layer is deposited vertically. One of the junction electrodes overlaps the Ta rectangle by $1 \mu \mathrm{m}^{2}$. For the single-tunnel devices (Fig. 2), the second electrode has a $3 \mu \mathrm{m}^{2}$ contact area with the Au pad. The diffusion engineered spectrometer has the right-hand electrode finished with a specific length narrow wire deposited at the same time with the junction. The wire ends in a $3 \times 5 \mu \mathrm{m}^{2}$ area that overlaps the Au contact.

When a photon is absorber in the left electrode, the excess created quasiparticles relax to near the $225 \mu \mathrm{eV} \mathrm{Al} \mathrm{gap} \mathrm{within}$ a couple of microseconds. The $700 \mu \mathrm{eV}$ Ta energy gap acts as a barrier, or a plug, impeding the quasiparticle diffusion to the left. The confined quasiparticles tunnel across the voltage-biased junction, each transferring an electron (negative) charge from left to right. They diffuse out into the Au pad in the case of the single-tunnel devices, while for the diffusion-engineered case the out-diffusion is hampered by the narrow wire. The idea of backtunneling due to geometrical confinement originated from $\mathrm{x}$-ray experiments with STJs [6]. If, due to a slow out-diffusion, quasiparticles dwell in the right electrode for a time longer than the tunneling time from the right to the left electrode, they will backtunnel. Since they cool during this time, reverse tunneling
TABLE I

Simulated OUTDIFFUSION Times AND AsSOCIATED MULTIPLICATION FACTORS

\begin{tabular}{|c|c|c|c|}
\hline Sample & $\begin{array}{c}\text { Wire geometry } \\
\left(\mu \mathrm{m}^{2}\right)\end{array}$ & $\begin{array}{c}\text { Pulse fall time } \\
(\mu \mathrm{s})\end{array}$ & $\begin{array}{c}\text { Charge multiplication } \\
\text { factor }\end{array}$ \\
\hline \hline \multicolumn{4}{|c|}{$\tau_{r e c}=150 \mu \mathrm{s}$} \\
\hline A & 0 & 3 & 1 \\
B & $0.25 \times 62$ & 18.8 & 6.3 \\
C & $0.25 \times 125$ & 37.7 & 12.6 \\
\hline \hline \multicolumn{4}{|c|}{$\tau_{r e c}=20 \mu \mathrm{s}$} \\
\hline A & 0 & 3 & 1 \\
B & $0.25 \times 62$ & 11 & 3.7 \\
C & $0.25 \times 125$ & 16 & 5.3 \\
\hline
\end{tabular}

is suppressed [6]. Backtunneling is a process that transfers a negative charge in the same direction as the initial (left to right) electron tunneling process. The confined quasiparticles continue to tunnel and backtunnel until they all diffuse out or recombine. Thus, the initially created charge is multiplied by a factor $\mathrm{p}$ proportional to the ratio of the confinement time, $\tau_{\text {conf }}$ to the tunneling time, $\tau_{\text {tunn }}$.

The experimentally observed tunnel time for our devices agrees with the theoretical prediction. For estimating the outdiffusion time, we model the diffusion in our device using a 2-dimensional diffusion equation. This is a valid approach for two reasons: the thickness of our films is much smaller than the lateral dimensions of our detectors, and the diffusion time over the thickness of our junction layers is much smaller than the other time scales. Using Matlab, we simulate the time it takes an initial narrow Gaussian distribution of quasiparticles created in the electrodes to diffuse out a narrow wire. The result depends on the diffusion constant of the material $D$, the quasiparticle recombination time $\tau_{r e c}$ and the wire dimensions. The loss mechanism is assumed to be uniform within the electrodes and the wire. Previously [7] we found that a good approximation to the 2-D simulation is the steady-state 1-D diffusion result, where the outdiffusion time is:

$$
\tau_{\text {out }}=A_{\text {effective }} \times \frac{L}{w} \times \frac{1}{D}
$$

where $A_{\text {effective }}$ is the effective $\mathrm{Al}$ area, $L$ and $w$ are the wire length and width, and $D$ is the quasiparticle diffusion constant in Al. This result diverges from the 2-D simulation as the time to reach steady-state in the wire becomes longer. Simulation results for $\tau_{r e c}=150 \mu \mathrm{s}$ and $20 \mu \mathrm{s}, D=7.5 \mathrm{~cm}^{2} / \mathrm{s}$ and different wire dimensions are presented in Table I. We extract the quasiparticle density in our devices from the measured subgap current. For a recombination constant $R$ of $3.36 \mu \mathrm{m}^{3} / \mathrm{s}$ [8], we expect a recombination time of $300 \mu \mathrm{s}$ at a temperature of $300 \mathrm{mK}$. A $20 \mu \mathrm{s}$ recombination time requires a recombination constant an order of magnitude larger. Previous measurements of the diffusion constant for pure $\mathrm{Al}$ in its normal state $D_{n}$ found a value of $60 \mathrm{~cm}^{2} / \mathrm{s}$ [9]. In the superconducting state, this value is reduced to:

$$
D_{q p}=\sqrt{\frac{2 k_{B T}}{\pi \Delta}} D_{n}
$$

where $D_{q p}$ is the quasiparticle diffusion constant, $T$ the effective temperature of the photon-created quasiparticle distribution, and 


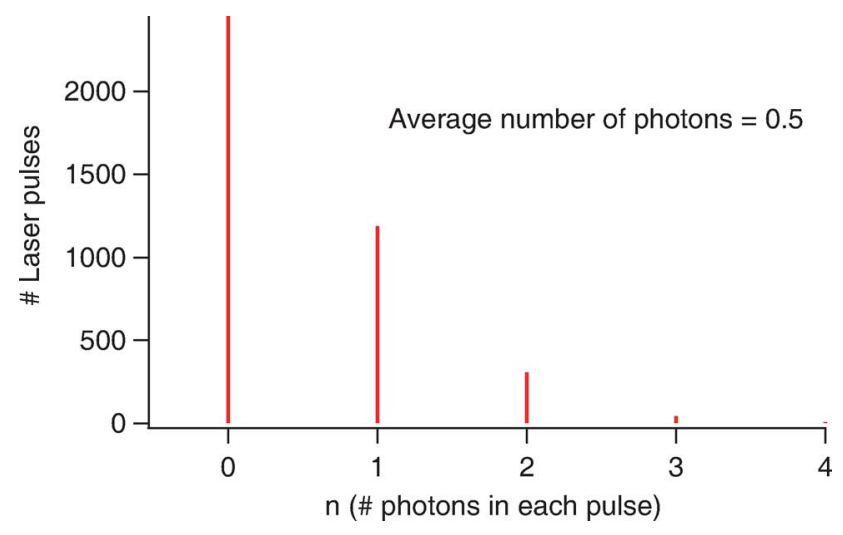

Fig. 3. Ideal Poisson distribution of photons with an average value of 0.5 .

$\Delta$ the superconducting energy gap. Samples B and C test the case of large out-diffusion time and achieves a large multiplication factor. We can lower the diffusion constant of Al by evaporating it in the presence of oxygen. We use $0.4 \mathrm{sccm}$ flow of oxygen during the $\mathrm{Al}$ evaporation to lower its normal diffusion constant. From RRR measurements of structures similar to the out-diffusion wires, we infer a value of $28 \mathrm{~cm}^{2} / \mathrm{s}$ for $D$ in the normal state.

We measured the response of our devices to UV photons from a pulsed $\mathrm{N}_{2}$ laser emitting photons at $3.68 \mathrm{eV}(337 \mathrm{~nm})$. The photons are guided by an optical fiber from the room temperature laser through a vacuum-tight feed-through to the cold stage of our double stage closed-cycle ${ }^{3} \mathrm{He}$ system. A set of neutral density filters, located on an optical bench between the laser and the dewar, is used to attenuate the light intensity. Each laser pulse lasts less than $4 \mathrm{~ns}$, and can be repeated at a maximum rate of $30 \mathrm{~Hz}$. The pulse width is much shorter than the quasiparticle diffusion time inside the electrodes, allowing us to use multi-photon absorption for simulating our detector's response to a single higher-energy photon. For a given attenuation value of the laser intensity, the number of photons in each pulse varies, obeying a Poisson distribution (Fig. 3). The probability of having $n$ photons in a pulse given that on average there are $\bar{N}$ photons per pulse is:

$$
P(n)=\sum_{n=0}^{n=\infty}\left(\frac{\bar{N}^{n} e^{-n}}{n !}\right)
$$

Data is recorded by a $20 \mathrm{MHz}$ digital oscilloscope Nicolet Integra 40 . We trigger our pulses on an optically-synchronized TTL laser output signal. Each pulse is digitized into 2000 points, with the first 500 points being the pre-trigger signal. Each data set used for constructing a histogram contains 4000 pulses.

For a noiseless detection technique, we expect the charge histogram of a set of pulses to obey the same Poisson distribution as the number of absorbed photons. But for every detected charge $Q$, the noise in our system introduces a charge uncertainty. Measuring many pulses generated by the absorption of the same number of photons, we build a charge histogram centered around an average charge, with an associated standard deviation that fits all the peaks in the multi-photon histogram. The conversion from the read-out charge to the photon energy is made via the device responsivity. We infer the energy deposited in the absorber from the average charge, and the energy

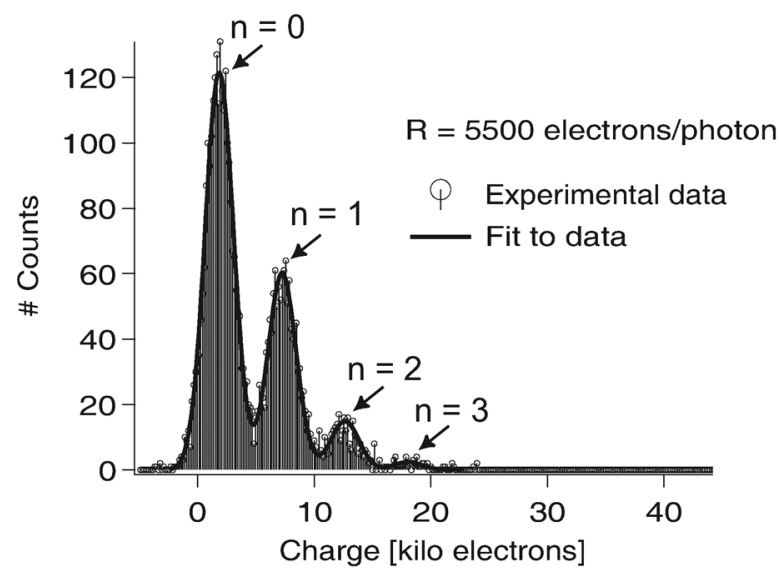

(a)

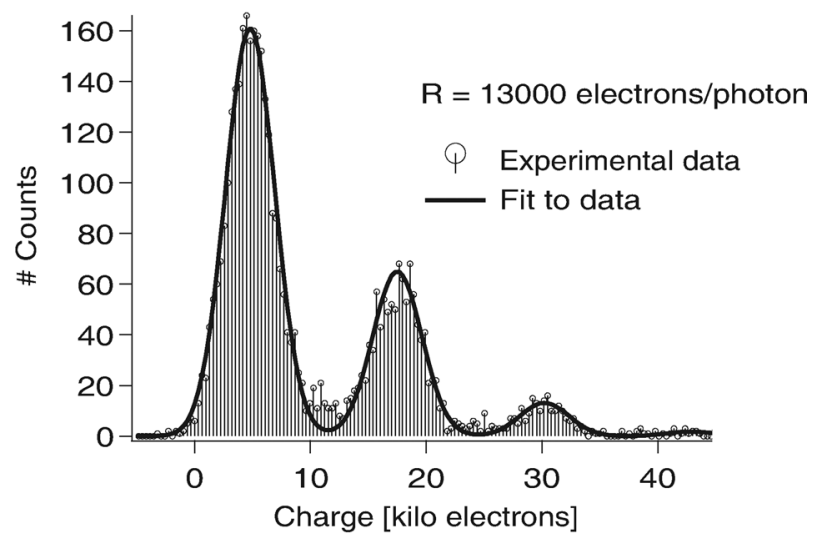

(b)

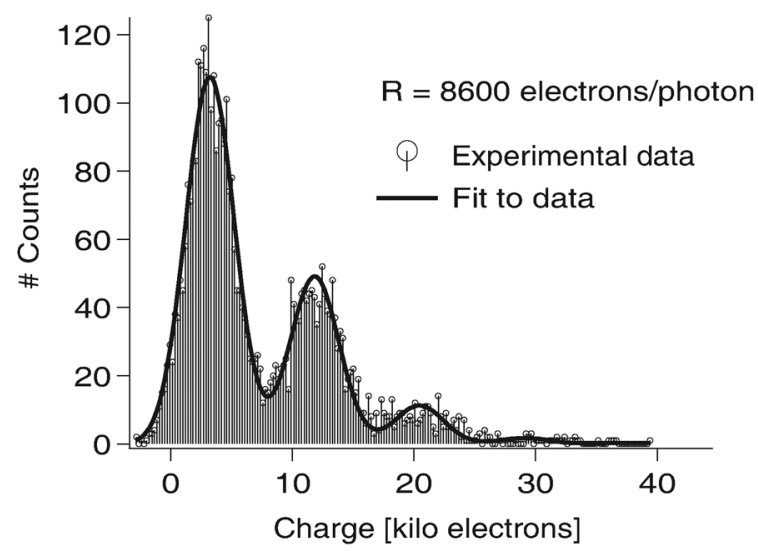

(c)

Fig. 4. Pulse histograms from single-tunnel and diffusion-engineered devices. a) Single-tunnel device; b) diffusion-engineered device (short outdiffusion wire); c) diffusion-engineered device (long outdiffusion wire).

resolution of our measurement from the FWHM of the charge histogram.

Our detector does not have an optical mask. Thus, increasing the number of photons that land on the absorber also increases the number of photons that land on the device's wiring and on the substrate, which cover a much larger area than the absorber. For each photon landing on the absorber, we have about $10^{4}$ photons landing outside of it. These photons create substrate photons which couple to the detector and break more Cooper pairs, creating excess noise. So for every event 
TABLE II

EXPERIMENTAL RESULTS

\begin{tabular}{|c|c|c|c|}
\hline Sample & $\begin{array}{c}\text { Pulse fall time } \\
(\mu \mathrm{s})\end{array}$ & $\begin{array}{c}\text { Charge multiplication } \\
\text { factor }\end{array}$ & $\begin{array}{c}\text { Energy resolution } \\
{[\mathrm{eV}]}\end{array}$ \\
\hline $\mathrm{A}$ & 4.3 & 1 & 1.8 \\
$\mathrm{~B}$ & 9.1 & 2.4 & 1.2 \\
$\mathrm{C}$ & 6.8 & 1.6 & 1.7 \\
\hline
\end{tabular}

we trigger on corresponding to zero photons landing on our absorber, there is a non-zero number of photons landing outside the absorber. Some of those photons land close enough to the junction to create substrate phonons that break Cooper pairs, creating a spurious pulse with a finite charge. We call this the offset charge. In Fig. 4 we present pulse histograms from 3 devices with the dimensions described in Table I. The results are summarized in Table II. The three histograms correspond to an average number of $0.51,0.40$, and respectively 0.46 absorbed photons. The responsivity (number of electrons per photon) and pulse fall times differ. Device A has a responsivity of 5500 electrons/photon and the pulse obtained from averaging all the pulses in the histogram has a characteristic fall time of $4.3 \mu \mathrm{s}$, close to the expected tunnel time. Device B has an almost double responsivity of 13000 electrons/photon and an average pulse fall time of $9.1 \mu \mathrm{s}$. The charge multiplication factor $p$ is 2.4 , while the ratio $\tau_{\text {out }} / \tau_{\text {tunn }}$ is 2.1 . Device $\mathrm{C}$ was tested in different circumstances. An Au film was deposited on a whole glass slide except in a central $0.4 \times 1.6 \mathrm{~mm}^{2}$ area. This Au mirror intended to reflect off the incoming photons was set about $1 \mathrm{~mm}$ above the absorber. The area exposed to photons around the absorber was thus halved. The region within a $30 \mu \mathrm{m}$ radius from the device and outside a $170 \mu \mathrm{m}$ radius were left exposed. With this setup we find a lower responsivity of 8600 electrons/photon and a average pulse decay time of $6.8 \mu \mathrm{s}$. This behavior indicates the existence of quasiparticles losses in the $\mathrm{Al}$ film of this device on a time scale of several tens of microseconds. A recombination time of $20 \mu \mathrm{s}$ agrees with our results for device B. New models including additional losses associated with the wire are necessary for explaining the short pulse decay from device $\mathrm{C}$.

We took data for several light intensities. The best energy resolution of $1.2 \mathrm{eV}$ was achieved using the short backtunneling device, $33 \%$ better than with the single-tunnel device. We ob- serve that the energy width increases with the absorbed number of photons for all three devices. We believe this is related to our multi-photon testing technique. Energy width is partly due to the statistical variation of the number of photons landing outside the absorber.

\section{CONCLUSION}

We successfully implemented our diffusion engineering approach for enhancing the energy resolution of single-photon spectrometers based on STJs in the UV energy range. We will further investigate the causes which limit our improvement in energy width compared to the single-tunnel devices. Fabricating and testing devices with different diffusion constants of the $\mathrm{Al}$ film will provide information regarding losses in the out-diffusion wire and their dependence on material purity.

\section{ACKNOWLEDGMENT}

The authors would like to thank R. J. Schoelkopf, R. Grober, and A. E. Szymkowiak for useful discussions.

\section{REFERENCES}

[1] N. Rando, S. Andersson, B. Collaudin, F. Favata, P. Gonodin, A. Peacock, M. Perryman, J. Verveer, P. Verhoeve, and D. J. Goldie, "First astronomical results from S-Cam," Nucl. Instr. and Meth. A, vol. 444, pp. 441-444, 2000

[2] Carl Zeiss, Inc., "META Laser Scanning Microscope 510 Data Sheet".

[3] C. M. Wilson, L. Frunzio, and D. E. Prober, "Superconducting tunnel junction detectors for extreme ultraviolet applications," IEEE Trans. Appl. Superconductivity, vol. 13, no. 2, pp. 1120-1123, 2003.

[4] P. Verhoeve, R. den Hartog, A. Kozorezov, D. Martin, A. van Dordrecht, J. K. Wigmore, and A. Peacock, "Time dependence of tunnel statistics and the energy resolution of superconducting tunnel junctions," J. Appl. Phys., vol. 92, pp. 6072-6081, 2002.

[5] G. J. Dolan, "Offset masks for lift-off photoprocessing," Appl. Phys. Lett., vol. 31, no. 5, pp. 337-339, 1977.

[6] L. Li, L. Frunzio, C. M. Wilson, and D. E. Porber, "Quasiparticle nonequilibrium dynamics in a superconducting Ta film," J. Appl. Phys., vol. 93, pp. 1137-1141, 2003.

[7] V. Savu, L. Li, A. Mukherjee, C. M. Wilson, L. Frunzio, D. E. Porber, and R. J. Schoelkopf, "Diffusion-engineered single-photon spectrometer for UV/visible detection," Nucl. Instrum. Meth. A, vol. 520, pp. 237-239, 2004

[8] C. M. Wilson and D. E. Prober, "Quasiparticle number fluctuations in superconductors," Phys. Rev. B, vol. 69, 2004.

[9] S. Friedrich, K. Segall, M. C. Gaidis, C. M. Wilson, D. E. Prober, A. E. Szymkowiak, and S. H. Moseley, "Experimental quasiparticle dynamics in a superconducting, imaging x-ray spectrometer," Appl. Phys. Lett., vol. 71, pp. 3901-3903, 1997. 